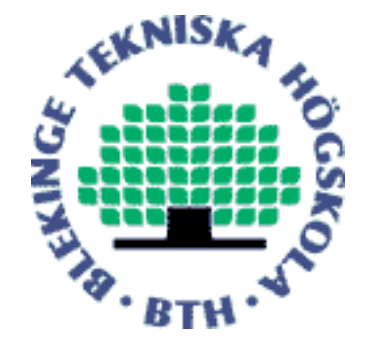

Copyright (C) 2013 IEEE.

Citation for the published paper:

Expectations and Achievements: A Longitudinal Study on an Offshoring Strategy

Darja Šmite, Daniela S. Cruzes

Symposium on Empirical Software Engineering and Measurement (ESEM)

2013 Baltimore

This material is posted here with permission of the IEEE. Such permission of the IEEE does not in any way imply IEEE endorsement of any of BTH's products or services Internal or personal use of this material is permitted. However, permission to reprint/republish this material for advertising or promotional purposes or for creating new collective works for resale or redistribution must be obtained from the IEEE by sending a blank email message to pubs-permissions@iee.org.

By choosing to view this document, you agree to all provisions of the copyright laws protecting it. 


\section{Expectations and Achievements: A Longitudinal Study on an Offshoring Strategy}

\author{
Darja Šmite \\ Blekinge Institute of Technology \\ Karlskrona, Sweden \\ darja.smite@bth.se
}

\author{
Daniela S. Cruzes \\ NTNU, SINTEF \\ Trondheim, Norway \\ dcruzes@idi.ntnu.no
}

\begin{abstract}
Offshore software development has gained momentum and most of software companies today have implemented offshore strategies of some sort. Many of these strategies are enforced by corporate top management and driven by assumptions that lower development wages guarantee cheaper and better software development. In practice, offshore software development is associated with many risks, and achievement of the expected benefits is not as straightforward as the rumor has it. In this paper we explore an implementation of an offshore strategy in a Swedish software company that opened its offshore branch in Russia. Based on extensive documentation analysis we create an overview of the initially expected benefits and obstacles that prevailed among onshore product and development unit managers. Years after implementation of the offshore insourcing strategy we asked these managers about the achievement of their expectations. We observed that the company documented various expected benefits when implementing an offshoring strategy and also concerns that some of these benefits might not be achieved. Seven years after its implementation, the offshoring strategy was overall considered working, however the expected benefits were not fully achieved. More importantly, several gaps were identified, that suggest that the enforced strategy has resulted in a stable but not beneficial collaboration from the onshore perspective.
\end{abstract}

Keywords - Offshoring, Insourcing, Global software development, Expected benefits, Perceived benefits, Concerns, Empirical case study, Longitudinal study

\section{INTRODUCTION}

Contemporary software engineering is trending more and more towards globally distributed collaborations, in which software engineers working on the same project are spread across multiple locations, often thousands of kilometers and several time zones away from each other. The popularity of global software engineering (GSE) is fueled by the rumors of significant cost savings. Software companies are attracted by the perceived benefits of cheaper, faster and better development [1] and hence offshoring today is practiced not only by multinational collaborations, but also by small and medium enterprises [2]. Despite its popularity, a systematic literature review on empirical evidence in global software engineering indicates that there are more problem reports than success stories [3]. An experienced reader of research material in the field, however, would most likely be accustomed to varying opinions ranging from significant success to total failure. For example, in an industry report Burger claims that his company saves several millions of euros per year [4], while lessons learned from a multiple-case study conducted at three Scandinavian companies suggest that first outsourcing contracts in all cases failed due to low productivity, poor quality and other collaboration troubles [2].

One of the reasons for the varied opinions on the phenomenon is that the analysis of success or failure in global software engineering in general is not a straightforward task. Although it is often referred to as one research field, GSE relates to a variety of different types of software development projects [5]. The differentiating attributes of these projects have been captured in a recent taxonomy [6] and include: location (onshore versus offshore), legal entities (outsourcing versus insourcing), geographic distance (close versus distant for onshore projects, and near versus far for offshore projects), as well as temporal distance (similar versus different for onshore projects, and small or large for offshore projects). Notably onshore projects (those distributed within one country) are often included as a type of global projects, while they can hardly qualify to be global in its common sense. Because global projects are enabled through a number of diverse scenarios, it is fair to assume that what works in one context does not necessarily apply in another [7]. Therefore deriving average success rates or cost saving rates from heterogeneous samples, as proposed in [8], is meaningless.

In addition to varying contexts, success or failure of GSE projects can be measured differently. In a systematic review, on dispersion, coordination and performance in global software teams, the authors identified 28 papers on empirical studies in the area [9] and concluded that all of the studies that used perception-based measurement of performance (for example, interviews with developers about performance of global projects) show a negative or neutral impact of geographical and temporal dispersion on performance. While perception-based studies report a pessimistic view of the impact of dispersion on team performance, studies on direct measurement show positive results.

Another essential aspect of measuring success of global software projects is related to the time perspective. Carmel and Tjia [10] attempt to systemize evaluation of economic benefits related to implementing an offshore strategy. Their approach suggests that it takes time for the economic benefits to realize as the "breakeven" is reached when the sum of direct cost savings reaches the sum of initial investments. It is fair to assume that the same rationale applies to other benefits, such as 
increased performance, innovative capabilities and faster development, to name a few. This means that short-term estimates of realization or non-realization of the expected offshore benefits might differ from the long-term estimates and thus lead to misleading conclusions.

Our objective in this paper is to provide an in-depth analysis of a longitudinal case study of implementation of an offshore insourcing strategy where we explore the realization and non-realization of the offshore benefits over a period of seven years from the point of implementation of the GSE strategy. In this case study we focus on the time aspect of the approach, and in the fact that the real effects of the offshoring approach are more perceptible after years of implementation of the strategy. Besides, we focus on the triangulation of the data sources and stakeholders of the offshoring strategy; the study is based on document analysis recorded in the beginning of the strategy development, documented implementation of offshoring, interviews made seven years after, and observations that were gathered regularly during two and a half years after the interviews. Our empirical study is driven by the following research questions:

RQ1: What were the initial expectations of benefits and obstacles of the onshore management for the introduction of an enforced offshore strategy?

RQ2: How onshore personnel perceived the achievement of the benefits of the enforced offshore strategy and obstacles for their achievement seven years after its implementation?

RQ3: What can be concluded regarding success or failure of the studied collaboration from comparing the initial expectations and their perceived achievements?

The rest of the paper is organized as follows. In Section II we discuss the expectations organizations have when initiating an offshoring strategy and studies discussing the success and failure to achieve these initial expectations. We pay specific attention to how and when the results are evaluated. Our research goals, objectives, research questions and methodology are outlined in Section III. Section IV contains results of our empirical study, which we discuss in Section V. Finally Section VI concludes the paper with a summary and implications for future research in the field of global software engineering.

\section{RELATED WORK}

GSE researchers have been engaged in the chase of evidence behind success or failure of global software engineering for more than a decade now. Some of them, such as [1] and [2], apply exploratory case study approach, while others, such as [8] mentioned above, aggregate the data from many projects with the help of a survey instrument. There are also secondary studies, such as [3] and [9], that aim at collecting and synthesizing evidence from existing empirical studies. In this section we discuss findings and approaches applied in the mentioned research studies, which serve as a motivation for our research.
Moe et al. in [2] discuss their findings from an empirical investigation of three Scandinavian small and medium software companies failing in their offshore outsourcing collaborations to Asia and further turning to offshore insourcing instead. Although in all three cases offshore outsourcing was driven by the cost reduction strategies and inability to employ locally, the failure in this study is associated with poor quality, decreased productivity and various other obstacles that finally led the outsourcing contracts to cancellation. In one of the failed collaborations the authors also mention that the expected cost savings were not achieved. Notably, one out of three companies studied decided to terminate the offshoring contract after the first year of collaboration. The other two companies invested into attempts to make their collaborations work, but after three years gave up and the offshore outsourcing relations were terminated. The authors suggest that though the failure of outsourcing could not be undoubtedly concluded, several important obstacles were associated with the global strategy studied, in particular, offshore outsourcing from small or medium companies to large offshore vendors.

Ó Conchúir et al. in an empirical investigation of three Irish branches of global software companies working with offshore sites around the world called "Global Software Development: Where Are The Benefits?" demonstrated that the expected offshore benefits are neither clear-cut nor can their achievement be taken-for-granted [1]. In all three cases the companies started global software development primarily to reduce costs. However, the authors examined the achievement or non-achievement of different frequently cited benefits. The authors concluded that reduced development costs, cross-site modularization of work, access to large skilled labor pool and closer proximity to market were only partially achieved, while leveraging time-zone effectiveness and innovation and shared best practice were regarded as mythical benefits. Notably, it is stated that the study is based on twelve semi-structured qualitative interviews with people with experience of establishing and managing several offshore teams, however, the exact time frame in the analysis remains unclear.

Similarly a study of offshore outsourcing in Schlumberger [11] indicates that the high expectations of immediate cost savings were not met because of significant managerial overhead, and that companies may have unrealistic expectations to start with. The author describes a learning process of the company that failed with initial offshore outsourcing of knowledge-intensive work and later adjusted the strategy. Notably, the findings indicate that it takes time to harvest the benefits. The author presents a three-stage model comprising a setup stage, a ramp-up stage and finally a mature stage, which is characterized by cost-effective productivity and satisfactory quality. Offshore outsourcing in this case also helped the company achieve the desired flexibility in resources without increasing the fixed headcount.

Finally, while the majority of companies start offshoring to reduce development costs, other explicit or implicit expectations exist. A number of studies emphasize the importance of defining and communicating these expectations. On the basis of a systematic literature review targeting solutions to various problems of managing distributed development projects Gomes and Marczak suggest that it is 
essential to communicate expectations and establish overall rules for working together to succeed [12]. Findings from an empirical investigation of a number of diverse distributed collaborations in Nokia suggest that defining and communicating expectations has a profound effect on project performance [7]. Furthermore, Barney et al. [13] have also found that undefined quality requirements create ambiguity as to what is expected and subsequently often mean that management expectations are not met. For a similar reason Jensen et al. [11] suggest defining key metrics that cover performance targets (quality and productivity) and providing regular and constructive feedback to enable opportunities for improvement.

In summary, although many companies are involved in offshore strategies of some sort, it is evident that offshore collaborations are not as successful as one may assume. This is why the rationale of starting offshoring "because everybody does it" might be dangerous. Long-term collaborations do not necessarily determine the success of an offshoring strategy. Unfortunately, the current GSE research neither provides sufficient illustration of the rationale behind the expected benefits to be achieved through offshoring, nor does it help to trace the long-term outcomes of offshoring to the initial expectations of the companies.

\section{RESEARCH APPROACH}

Addressing the challenges discussed in the previous sections we performed a longitudinal exploratory empirical case study of an offshoring strategy as seen and experienced by the company onshore employees. Longitudinal nature of the study is motivated by the duration of the case (an offshoring strategy) as commonly interpreted in software engineering [14], in combination with the extensive period of time over which our study took place. In particular, we illustrate the process of how a company defined initial expectations from implementing an offshoring strategy, benefits and associated concerns, and later we demonstrate their perception of achievement or non-achievement of the benefits and concerns associated with offshoring. The longitudinal data collection was done partially retrospectively and partially through direct data collection and observations. We also reflect on the lessons learned from performing this type of research to improve the validity, generalizability, and comprehensiveness of the future empirical research.

\section{A. The Dolphin Case}

Dolphin $^{1}$ is a medium-sized business unit owned by a major multinational corporation producing software intensive products with the main development centers located in Sweden and corporate management situated in the US. Although software development is not the company's primary business, the amount of software embedded in its products grows every day. For cost-saving reasons primarily the corporate management decided to enforce the implementation of an offshore development in 2004. Employment in the high-

1 The pseudonym of the company initially appears in [2] and is reused in this paper for ability to synthesize the findings, if needed cost countries was restricted and the central development site in Sweden was thus forced to optimize their project portfolio to achieve full business potential and balance high-cost resources with those in low-cost regions. Similarly to many other companies Dolphin's management introduced a quota for how many resources shall be situated offshore $(70: 30 \%$ rule), while the choice and implementation of the actual offshoring strategy was left for the local Swedish management to be decided.

The implementation of an offshoring strategy started with a definition of goals and expectations for the strategy. In one of Dolphin's documents the managers mentioned:

"The vision for our global software engineering effort is to be much more competitive at developing outstanding software solutions than our competition. This requires us to be outstanding at identifying needs and opportunities in the markets, converting these into software architectures and specifications, and finally developing, testing and delivering the product in a cost effective and timely manner" (source: from a historical document).

Many meetings were organized, followed by a careful analysis of different locations, and a strategy was traced for following up the first years of transference of the development of the products to the new site. It was decided to keep the highprofile jobs related to identifying needs, creating high level specifications, validating the correspondents of the developed products with specifications and managing the overall projects in the high-cost countries, while the majority of the software implementation was decided to be relocated to the new offshore site in Russia. In 2011, three out of seven major products were developed in Russia with new projects being initiated. The offshore site employed 19 developers, while there remained only four developers onshore in the main site in Sweden.

The team responsible for the GSE approach established the following steps (among others) in a plan for investigating the approach:

- Develop software business model,

- Develop intellectual property rights (IPR) strategy,

- Develop process and organization,

- Set up facility and correct legal structure in Russia,

- Perform an analysis of the products that Dolphin was working with from an offshoring perspective.

For each potential product to be offshored, a thorough analysis was performed and documented by the company. These documents aimed to answer the following questions to provide the ground for making informed decisions:

- How large is the product? How many developers work on the product today?

- How modular is the product today? Are there welldefined interfaces? Identify the dependencies on other products, if any. How could the product be split up (technically) for offshore development?

- What types of competence are required for developing the product? 
- Which tools are required for the development environment? Is special hardware required?

- How well is the product documented? Which types of documents exist?

- Are there variations in the product that result in separate products (increased complexity)? Is the product still undergoing major new development or is it mostly in maintenance state? Are future plans currently known for the product?

\section{B. Data collection and analysis}

The collected research data (see Figure 1) that we used to answer the research questions were based primarily on document analysis and interviews. Additionally, we have used observations that were gathered on a regular basis during two and a half years after the interviews, as a part of the three years long research collaboration, as well as emails that we exchanged with the company representatives to verify the information about the cause of events and current characteristics of the organizational setup.

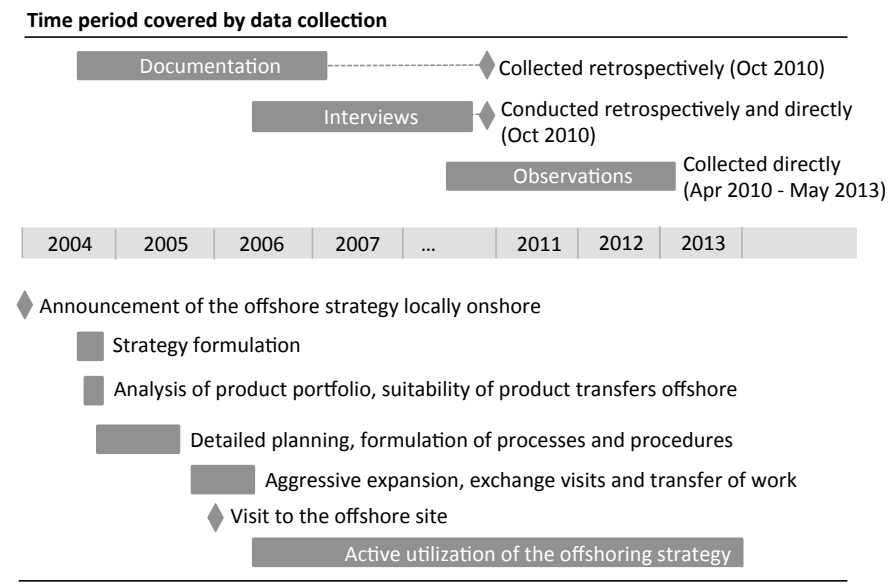

Events related to offshoring strategy

Fig. 1. Data collection

\section{RQ1: Initiation | 2004-2007 | Document analysis}

To answer the first research question we have collected data regarding initial expectations of the company from implementation of an offshore insourcing strategy. This was done by collecting company documentation, including internal presentations, meeting minutes, offshore risk analysis reports, product analysis reports, and new offshore insourcing implementation documents. As shown in the timeline of the offshore strategy at Dolphin (see Figure 2), many documents were generated, especially in 2004, 2005 and 2006, on the implementation process and also on the initial results of the strategy. In total 53 documents were collected for further analysis. These were carefully reviewed by both authors together, and only documents that contained information about company's expectations regarding initiation and implementation of the offshore strategy and associated concerns were included for further analysis. This left us with 16 documents.

Qualitative data analysis based on open coding [15] and sensemaking techniques was performed by both authors together. Each document was read, discussed and coded. We applied open coding techniques, in which different categories emerged from the data. The categories were discussed and refined iteratively in the process of data analysis. The coded data was documented in a Mindmap tool called MindManager Mindjet. In total, 61 extracts were coded forming five final categories. These categories were all related to different expectations regarding implementation of the studied offshore strategy and were used to answer the first research question. Figure 4 shows the results of the coding process in detail. The figure illustrates branches of analysis with categories as nodes of the mind map tree. The sources of information (documents) were hidden for confidentiality reasons, but appeared as final leafs in the original mind map.

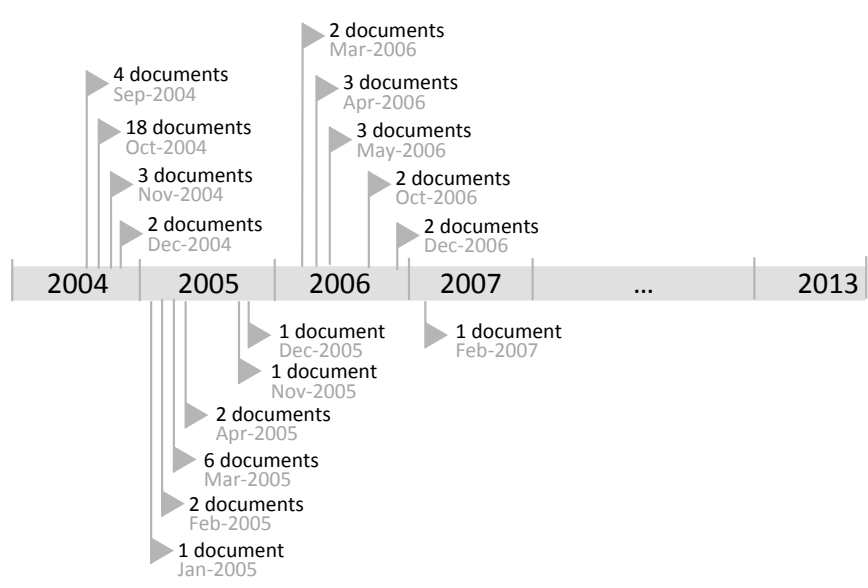

Fig. 2. Collected documents on the timeline

RQ2: Offshoring | 2007-2013 | Interviews and observations

To address our second research question we conducted semi-structured interviews with eight out of 18 onshore representatives involved in software development and especially product management. The interviews were conducted in person in October 2010 and took 45 min on average. Among interviewees were unit managers, product managers, and developers representing different projects, who were selected by the company to represent different projects and units working with offshore developers. During these interviews we asked questions regarding transition to offshore development, initial expectations and how these were monitored and measured, gains and losses associated with the transition and perceived characteristics of successful offshore projects (as described in the Appendix). All interviews were recorded and later transcribed, coded using open coding techniques, and documented using MindManager Mindjet. The coding resulted in 94 extracts coded under five final categories as shown in brief in Figure 3. Data collection was performed by the first author, while data analysis was done by both authors together. 
Additionally we used observations as an additional source of information. These were generated during regular research group meetings during April 2010 - March 2013 and during three days long visit of the offshore site in December 2011. Observations helped to supplement our results with other insights into the state of the offshoring collaboration after the interviews.

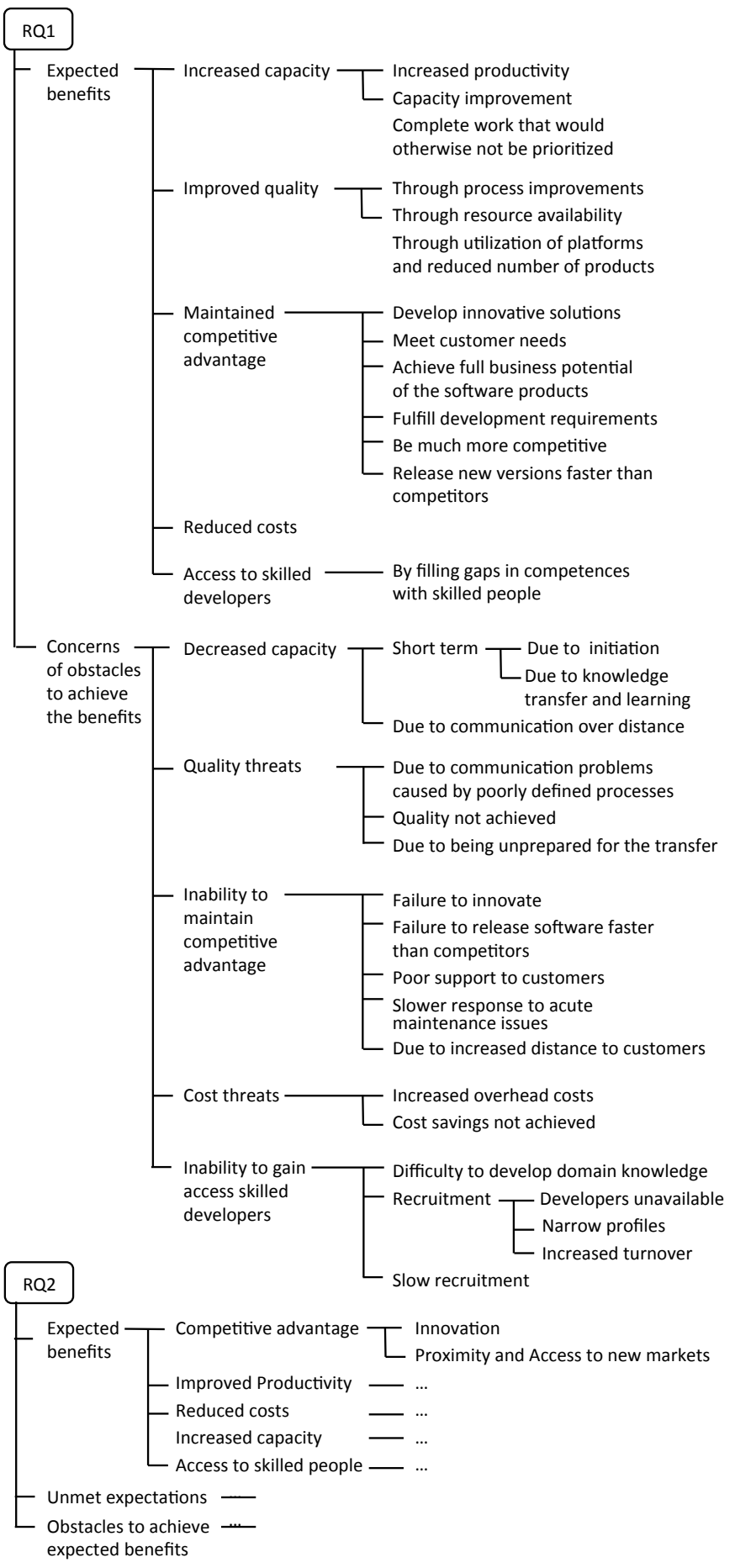

Fig. 3. Categories emerging in the data analysis

\section{RESUlTS}

In this section we present our results structured according to the research questions.

\section{A. Initially expected benefits and concerns of onshore management for the introduction of an enforced offshore strategy (RQ1)}

The results were grouped into expected benefits and concerns as described in the previous section.

Expected benefits: From the meeting minutes and initial presentations we learned that the company had defined goals and expectations for the forthcoming collaboration. These we have grouped into the following categories: increased capacity, improved quality, maintained competitive advantage, reduced costs and access to skilled developers (as illustrated on Figure 3).

The most desired and frequently mentioned benefit from implementation of an offshoring strategy was related to capacity improvement. This was associated with expectations of more timely delivery as a result of increased productivity. Offshoring was expected to finally provide dedicated welltrained developers that would be able to fulfill development requirements and thus customer needs that were impossible to fulfill due to the shortages in resources and competences.

Offshoring was also expected to lead to improvements in quality of the processes and outcomes. The company assumed that to start offshore development, internal processes would need to be standardized, and thus improved documentation, requirements specifications and other processes were expected to reach higher maturity levels. Besides, ability to employ professionals at a lower cost was seen as a strategic opportunity to fill the current gaps in competences.

Assuming that the above-mentioned benefits would be achieved, the company expected to maintain competitive advantage. In particular, the company expected to be able to develop innovative solutions, as well as to release new products and product versions to the market faster than their competitors.

Finally, offshoring was also associated with increased revenues and cost benefits that would ultimately help in increasing capacity and improving quality and competitiveness.

Concerns: Analysis of the documentation indicates that along with the expectations different concerns were attributed to the offshoring strategy implementation and discussed at various occasions. Interestingly and perhaps contradictory the expressed concerns match with the expectations. These were: decreased productivity, decreased quality, loss of competitive advantage, increased overheads and inability to recruit skilled developers (as illustrated on Figure 3).

The company feared that initiation of an offshoring strategy would negatively affect the productivity in short term due to implementation of a global organization, time needed for developing and establishing new processes and transferring 
the knowledge. It was also expected that communication over the distance and the effect of the learning curve could slow down the development.

Fear of an inability to maintain competitive advantage was associated with the possible failure to innovate and maintain the needed time-to-market. The company feared that customer support would suffer due to slower responses to acute maintenance issues, increased distance to customers.

The company also assumed a possibility that quality improvements might not be achieved. The risks here were associated with a difficulty of hiring staff according to requested profiles, challenges in developing domain knowledge, increased turnover of the offshore personnel and the loss of key onshore developers due to the introduction of offshoring.

Establishment of a new organization was also associated with the increased initial overhead. Since the company was not fully prepared for the new strategy, considerable work needed to be done, including translation of existing documentation, development of complete documentation of products and process descriptions. This was also seen as a risk. Besides, the company discussed potential failure to recruit developers with the needed expertise and reach the assumed cost savings.

\section{B. Onshore personnel's perceived achievements of the}

benefits and of the enforced offshore strategy and obstacles to achieve these benefits seven years after its implementation (RQ2)

As shown in Figure 3, from the interviews with the onshore personnel three main themes of discussion were extracted: expectations of the personnel towards the offshoring strategy, obstacles that prevent achievement of these expectations and discussions on the benefits that were not achieved.

Expected benefits not achieve: The most frequently mentioned expectation was clearly related to cost reduction. The interviewees mentioned that they believe cost is the main driver for the offshoring strategy. One interviewee mentioned:

"The company is extremely restrict of a headcount. So that means that that is regulated from the top. It is basically the sum of the simple calculation. They look what is your trade sales, what is your rate daily cost like, how is that involved in next year and then you get a headcount."

The interviewees said that they are not totally sure if there are any cost savings achieved. The common answer suggests that there is no belief in cost savings, but it has been also noted that these are difficult to measure taking all the aspects into account. One interviewee mentioned:

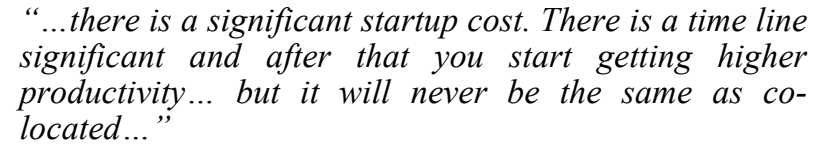

Increased competitive advantage and access to qualified people were the other two main expectations from implementation of the offshoring. On the increased competitive advantage, all the interviewees stated their outlook towards this expectation. One interviewee said:

"I would love that they (Russians) would come up with their own ideas. And it happens also but I wish it could happen more often. I think it is a good thing that people working with a product take their own initiatives and add value. I am transferring my knowledge about the users to them. But they don't get their own feedback very much from end users."

Also when asked if they thought they have achieved proximity to the market and the customers or new customers due to being present in the offshore location, they all answered that it did not happen. One interviewee said:

"No, in Russia ... the priority has been - cost savings.

We have some Russian customers but there is no infrastructure there, where we actually get those Russian customers in touch with us."

In respect to the access to qualified people, the interviewees expressed satisfaction with the skills of the people they were able to recruit in Russia. Besides, the turnover rate of the employees was low. One interviewee said:

"I think in general the people we have employed, the site management have employed, have been very good people with some exceptions. But, I mean, it is the same here. Sometimes you succeed sometimes you do not. I think that the education system in Russia is good and the universities are good. There is the output of welleducated engineers."

Half of the interviewees mentioned that increased performance was their initial expectation. However, all were in agreement that this benefit was not achieved in the organization. One interviewee said:

"I think if you will compare to Sweden you would have a more rapid organization if you would do it here. So you would shorten time-to-market basically. And I think you would also improve quality."

On the quality of the final product, the interviewees did not mention anything on the expectations to this aspect, but when asked explicitly on what were the benefits not achieved, three of them mentioned that the quality of the product concerned decreased after offshoring.

Obstacles to achievement of the expected benefits: During the interviews the onshore personnel mentioned some problems/obstacles that they faced during the seven years of the offshoring.

The interviewees frequently commented about the perception of loosing proximity and the group feeling, dependability and that they have more communication problems. One respondent said:

"The difference is that here you create a sense of belonging. Because here is where the management is, where we do the production. So there is a feeling of pride in this building that this is the business that we 
build up a lot of knowledge about people here, this is where the president sits, where the plans are made and so on. The offshore site, if you are not careful, it is a business hotel."

Another respondent said:

"If you could have everything at one place you would have this issue, to build a team spirit, so, it is easy to work together".

On the communication issues, one respondent said:

"Building up the competence in (Russia), due to communication, culture and language problems are not as quick."

Another obstacle mentioned by the respondents as a longterm problem caused by the offshore strategy was that the onsite personnel lose the touch with the technology and the technical aspects of the project. As one interviewee noted:

"I think we lose some knowledge also because actually we are loosing development experience. We might do the wrong decisions because we don't have any practical experience anymore."
Another interviewee emphasized:

"Sweden is too far from development".

And another interviewee said:

"I think that we lost the touch with the technology, we do not know really now what is new in the area. Another loss is the ability to support the rest of the organization. For instance, we do a production here and development there. When the production has a problem with configuring a software, there is nobody here who knows the technology. Then we always have to buy services or get somebody on the phone."

Related to the previous obstacle, the three interviewees said that sometimes the offshore site loses focus of the overall picture of the project. One interviewee described:

"In case of long time between releases it is difficult to keep people focused because people lose track of what is going on and developers are switched in the middle of a version."

On the productivity aspect, the respondents believed that the startup is quite long and that the whole onshore team spends too much time in monitoring and control.

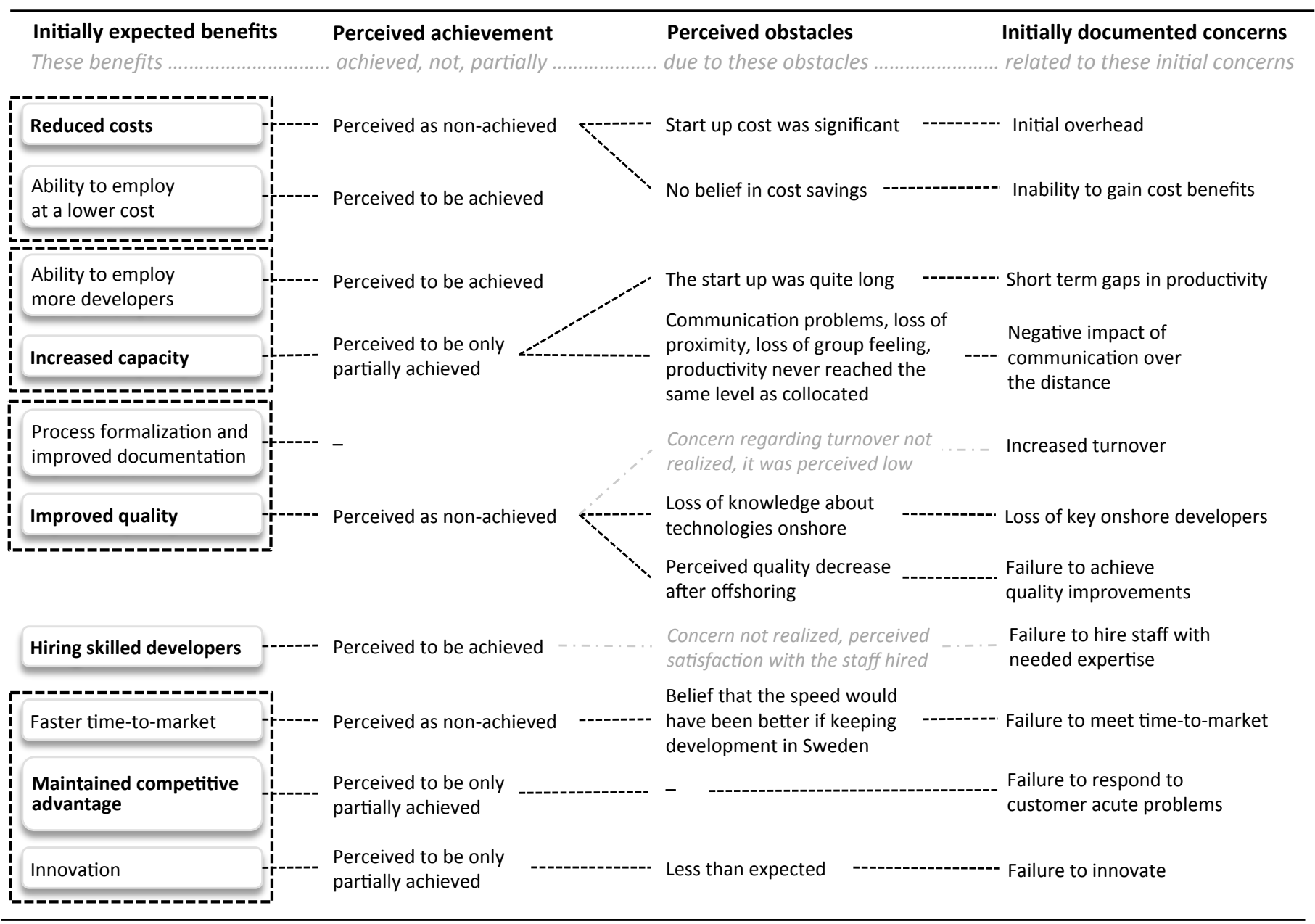

Fig. 4. Comparison of initially documented expectations and their perceived realization 


\section{Success or failure of the studied collaboration (RQ3)}

In this section we address the question of what can be concluded regarding success or failure of the studied collaboration from comparing the initially expected benefits and concerns (RQ1) and their perceived realization seven years into the collaboration (RQ2) (see Figure 4). The figure shall be read from the left to the right following each of the expected benefits. In this way we have summarized and highlighted the key findings that emerged in our analysis.

First of all, we observe that the onshore personnel do not believe that all initial expected benefits are achieved. Despite the general agreement that the corporate management has initiated offshoring purely for cost reduction reasons, there is no belief that the actual cost savings would have been achieved. One important point was that during the initial planning of the strategy we could find many aspects of improving quality of the product and process documentation to be expected, but in the interviews, it was an aspect that the respondents did not mention. More importantly, the overall attitude and obstacles mentioned indicate that expected improvements have not been achieved and some interviewees perceived that the quality even decreased after offshoring during the seven years.

Secondly, it is notable that some of the initial concerns surfaced and created obstacles to achievement of the expected benefits. The company was quite concerned with quality, productivity and especially with the recruitment of staff in the new site. However, the interviews indicate that the recruitment was not an issue and the interviewees perceived to be successful with hiring people in the Russian side. Turnover also appeared to be low against initial concerns. Productivity on the contrary was seen as an issue, because the Swedish side did not feel that they gained productivity. Besides the loss of performance during the startup, the interviewees believed that the teams constantly lose speed due to being distributed.

And finally, the responses of the interviewees suggest that some of the concerns were underestimated and in reality caused larger obstacles than initially anticipated. In particular, the people involved in the planning of the strategy did not foresee the obstacles related to alienation. Our interviewees complained about the feeling of being two separate entities, loss of teamness and the big picture in the product development. Also the loss of knowledge about the technology onshore was not at all expected.

\section{DISCUSSION}

The results of our study give insights to both industrial practitioners and researchers. For practitioners, the results of our longitudinal study confirm the view that software companies do have high expectations from implementing offshoring strategies, and like in other related studies these expectations are neither clear cut, nor can their achievement be taken for granted $[1,2,11]$. For researchers, our study shows that researchers should account for the complexity of the offshoring strategy and especially for the level of maturity of the strategy when interpreting the results of the study in order to make a credible industrial empirical study.

\section{A. Implications for industry}

The longitudinal perspective of the study and data collected at various points of time enables the traceability of the longterm effects of an offshoring strategy to its initialization. Similarly to other companies Dolphin's corporate management enforced offshoring and restricted the headcount. We observe that although the management responsible for implementing the strategy was positive about the initiative (this is supported by multiple improvements expected), they also had several concerns that could endanger their expectations, and put multiple mitigation strategies to address them (such as nearshore location and selective recruitment). We believe hat early risk management is the main reasons for why this collaboration worked out and is stable, as opposed to many other abandoned offshoring attempts as a result of a lack of preparation and overlooked threats $[2,10,16]$. We further see, however, that many of the Dolphin's initial concerns emerged during the implementation of the offshoring, creating obstacles to the achievement of the expected offshore benefits, which confirms the established view that offshoring is not an easy journey.

Because we could not elicit information from the corporate management we are unable to judge the rationale behind the offshoring decision. However, the available data suggests that to offshore or not to offshore was never a question. We also observe that salary savings and enforced headcount quotas are the main targets discussed by the onshore personnel in relation to the corporate management expectations, which in the Dolphin's case came on the expense of the quality, productivity and limited innovative capacity. Notably, onshore management seems to have foreseen many effects of the offshoring strategy in advance. We therefore suggest that corporate management in such cases shall involve onshore management representatives in offshoring decision process, as they may provide more realistic input regarding the potential threats and impacts of offshoring on the software development level.

It is important to emphasize that, although not all benefits have been achieved, the company studied has achieved a stable offshoring strategy, and invested in making their relationship work. On the run, some of the highlighted characteristics that lead to success of an offshoring strategy from the perspective of our interviewees are:

- Keep the people in the project involved in what's going on with the business and technology; and, have people on-site always in touch with the technology;

- Establish two-way mechanisms and infrastructure for knowledge transfer and knowledge sharing;

- Choose offshore places with insignificant temporal difference to enable direct communication;

- Introduce face-to-face meetings in critical parts of the projects;

- Employ skilled offshore personnel, fluent in English. This is important for staff in all roles, and not just the site management, to avoid bottlenecks and misunderstandings. Personally participate in the hiring process; 
- Account for differences in cultures. It is what people understand that counts, what is expected from you, what others expect you to do;

- Put in place strategies that encourage staff stability on the offshoring site.

\section{B. Implications for research}

Like Ó Conchúir et al. [1] we have found that reduced development costs, faster time-to-market and innovation and shared best practice were desired but not fully achieved. We also found that the onshore people perceived the quality to be lower than before offshoring. Notably, several interviewees referred to the initial start up stage, which was characterized by decreased productivity and quality as the offshore employees climbed the learning curve, which we relate to the ramp-up stage as suggested by Jensen et al. [11]. If our study had taken place during that stage, it is likely that the evaluation results would be more negative towards the fulfillment of the expectations to the strategy. During the time of the interviews, seven years after the initiation of the relationship, the interviewees perceived the relationship to be better than that during the start up stage, and there was a general acceptance that the relationship is working despite the challenges. However, we observe that the general perception is that productivity and quality requires further improvements. Thus, we suggest that the relationship has not reached the maturity. We find that the maturity stage model described by [11] is useful to check the evolution of an offshoring strategy, and may help the companies like Dolphin to monitor their relationships. In this way, we learned that offshoring in the Dolphin's case is not perceived as the best substitute to onshore development as the onshore personnel believes that the internal collocated development would help to achieve a better quality, faster time-to-market and possibly be more cost-effective at the end. We interpret these results as signs of skepticism in the rationale behind the offshoring decision enforced by the corporate management. If the further maturation of the strategy does not bring the desired levels of quality and productivity (which seemed to be more important from the points of view of the interviewees) it is not unlikely that unwillingness to collaborate, opportunism, self-protective behavior and distrust could emerge, as found in a similar case of an enforced offshoring reported in [17].

\section{Possible limitations of the study}

The main limitations of our study were caused by the nature of the industrial data and by our data analyses. There are a number of limitations to this study. First, qualitative findings are highly context- and case-dependent [18]. Three kinds of sampling limitations typically arise in qualitative research designs: cases that are sampled for observation (because it is rarely possible to observe all situations); time periods during which observations took place (problems of temporal sampling); and selectivity in the people who were sampled either for observations or interviews or in document sampling.

Our study was a longitudinal single-case holistic study. Although we could decide which data we wanted to collect and analyze, we could not influence the collection of the initial documents produced on the initial stages of the offshoring strategy. One possible threat to the trustworthiness of our data analysis is that we did not perform other document analysis of the period in between the initial stages of the offshoring strategy and the interviews seven years later.

Another possible threat to the trustworthiness of our data analysis is that the benefits of the strategy may have varied depending on the project, we did not account for the differences between projects, but to an overall perspective of the perceived views towards the strategies. According to the initial documents, the strategy would be performed in stages, from simpler maintenance projects to new development projects. It may be that the personnel have a more negative perspective if they were involved in the more complex projects. One way to overcome this limitation is that we interviewed people in Sweden that are in the company since before the offshoring strategy and were involved in different projects during the seven years, and each project was covered by several interviews.

Our study might be biased as a perception-based study according to [9], and have a more pessimistic view on offshoring than in reality. It also lacks the investigation of the results from the offshoring personnel; they may have different perceptions of the approach as well as of the benefits achieved. However, interview and survey data gathered from the offshore location in a related research study (unpublished) indicates that the offshore personnel is aware of productivity deficiencies, communication problems and other challenges of collaboration. On the onshore personnel we tried to involve the perspective of different roles in the projects: unit managers, product managers, and developers.

On the transferability/generalizability of the results of this study to other settings or groups: to promote transferability, we described the selection and characteristics of the case study and we focused a section on describing the context and settings, data extraction, and synthesis process in detail, as well as quotations with our major findings.

\section{CONCLUSIONS}

In this paper we discussed our findings from performing a longitudinal empirical study of expected offshore benefits and their perceived achievement in a Swedish company developing software-intensive products in collaboration with a Russian site of the company. We collected and analyzed documentation created in support of initiation of the offshoring strategy, as well as interviews with onshore site representatives conducted seven years after the implementation of offshoring.

Based on our results we conclude that although the offshoring strategy can be judged as stable and satisfactory, the initially expected benefits were perceived as only partially achieved. We have found that the company succeeded to employ skilled developers and more developers than have been employed onshore, and also gained salary savings. However, the development capacity was seen as only partially improved and the onshore personnel were skeptical regarding the true value of the cost reduction. More importantly, we found that onshore personnel perceived that the quality and development productivity was better before the offshoring, and thus 
competitive advantage of the company did not improve with the strategy, as expected.

In our analysis we have observed the differences between the short-term and the long-term effects of offshoring. This means that the real effects of the offshoring approach are more perceptible after years of implementation of the strategy.

Noteworthy we have studied a case of an enforced strategy, in which corporate management has introduced quotas for recruitment in high-cost and low-cost countries. The strategy implemented by the Swedish branch of the company has been a response to the corporate policy and measured by the headcount is obviously successful per definition. However, our findings indicate that a more versatile view on the achievement and non-achievement of expectations helps to assess the weaknesses and strength of the chosen strategy. Detailed analysis of initial documentation suggests that the onshore management could foresee many risks and thus we argue that corporate management shall elicit input from the local management when taking offshore decisions.

We have discussed the implications of the results for research and practice as well as the limitations of the study. For future works, we intend to investigate further the matching between the perceived view of the strategy between the offshore personnel versus the onshore personnel. It would be also interesting to further examine the definition of success in offshoring collaborations from different perspectives, e.g. corporate management's, onshore (originating site) and offshore (destination site).

\section{ACKNOWLEDGMENT}

We are sincerely thankful to all employees of the case company that were involved in this research. This research is funded by the Swedish Knowledge Foundation under the KKHög grant 2009/0249.

\section{REFERENCES}

[1] Ó Conchúir E, P Agerfalk, H H Olsson, and B Fitzgerald, "Global software development: where are the benefits?" In: Commun. ACM, 2009, 52(8): 127-131

[2] Moe N B, D Šmite, and G K Hanssen "From Offshore Outsourcing to Offshore Insourcing: Three Stories", In: Proceedings of the IEEE International Conference on Global Software Engineering (ICGSE), 2012, pp.1-10

[3] Šmite D, C Wohlin, R Feldt, and T Gorschek "Empirical Evidence in Global Software Engineering: A Systematic Review", In: Journal of Empirical Software Engineering, 2010, 15(1): 91-118

[4] Burger W "Offshoring and Outsourcing to INDIA," Global Software Engineering, In: Proceedings of the IEEE International Conference on Global Software Engineering (ICGSE), 2007, pp.173-176

[5] Šmite D, and C Wohlin "A Whisper of Evidence in Global Software Engineering”. In: IEEE Software, 28(4): 15-18

[6] Šmite D, C Wohlin, Z Galviņa, and R Prikladnicki "An Empirically Based Terminology and Taxonomy for Global Software Engineering", In: The journal of Empirical Software Engineering. Available online since 2012

[7] Poikolainen $\mathrm{T}$ and $\mathrm{J}$ Paananen, "Performance Criteria in InterOrganizational Global Software Development Projects", In proceedings of International Conference on Global Software Engineering (ICGSE), 2007, pp. 60-70
[8] Estler H-C, M Nordio, C A Furia, B Meyer, and J Schneider, "Agile vs. Structured Distributed Software Development: A Case Study," In: Proceedings of IEEE International Conference on Global Software Engineering (ICGSE), 2012, pp. 11-20

[9] Nguyen Duc A, D S Cruzes, and R Conradi, "Dispersion, coordination and performance in global software teams: A systematic review," In: Proceedings of an ACM-IEEE International Symposium on Empirical Software Engineering and Measurement (ESEM), 2012, pp.129-138.

[10] Carmel E, and P Tjia. "Offshoring Information Technology: Sourcing and Outsourcing to a Global Workforce”. Cambridge University Press, NY, 2005

[11] Jensen M, S Menon, L E Mangset, and V Dalberg, "Managing Offshore Outsourcing of Knowledge-intensive Projects - A People Centric Approach," In: Proceedings of the IEEE International Conference on Global Software Engineering (ICGSE), 2007, pp.186-196

[12] Gomes V and S Marczak, "Problems? We All Know We Have Them. Do We Have Solutions Too? A Literature Review on Problems and Their Solutions in Global Software Development," In: Proceedings of the IEEE International Conference on Global Software Engineering (ICGSE), 2012, pp.154-158

[13] Barney S, C Wohlin, P Chatzipetrou, and L Angelis, "Offshore Insourcing: A Case Study on Software Quality Alignment," In: Proceedings of the IEEE International Conference on Global Software Engineering (ICGSE), 2011, pp.146-155

[14] Runeson P, M Höst, R Austen, and B Regnell "Case Study Research in Software Engineering: Guidelines and Examples", Wiley, 2012

[15] Creswell J W "Research Design: Qualitative, Quantitative, and Mixed Methods Approaches", $2^{\text {nd }}$ edition, Thousand Oaks, CA: Sage, 2003

[16] DeLone W, JA Espinosa, G Lee, and E Carmel, "Bridging Global Boundaries for IS Project Success", In: Proceedings of the Annual Hawaii International Conference on System Sciences (HICSS), 2005, pp.48b

[17] Piri A, T Niinimäki, and C Lassenius, "Fear and distrust in global software engineering projects". In: J. Softw. Maint. Evol.: Res. Pract., 2012, 24(2): 185-205

[18] Patton M Q, "Enhancing the quality and credibility of qualitative analysis". Health services research, 1999, 34 (5 Pt 2): 1189-1208

\section{APPENDIX - INTERVIEW QUESTIONS}

Expectations:

What were the expectations to be achieved after implementing the decision?

Where these expectations achieved?

If yes, after how long? How was it measured?

Did you expect to:

- Maintain the same level of quality (provide the definition)

- Maintain the same level of performance (provide the definition)

- Maintain the same service level for the customers (provide the definition)

Benefits:

What were the expected benefits for implementing the decision?

Where these expectations achieved?

If yes, after how long? How was it measured?

- Reduced development costs

- Faster time to market

- Access to skilled labor pool

- Innovation and shared best practice

- Closer proximity to market and customers

- Increased number of customers

Are there any benefits that were not expected, but achieved?

Are there any lost benefits that were not expected, but were faced?

Could these benefits be achieved internally? How?

Success:

How would you characterize a successful offshoring project? 